\title{
ADAPTABILIDADE E ESTABILIDADE DE CULTIVARES DE MILHO PARA PRODUTIVIDADE DE MATÉRIA SECAE DEGRADABILIDADE RUMINAL DA SILAGEM
}

\author{
MAXIMILIAN DE SOUZA GOMES ${ }^{1}$, RENZO GARCIA VON PINHOㄹ, JACKSON SILVA E \\ OLIVEIRA $^{3}$, MAGNO ANTONIO PATTO RAMALHO ${ }^{4}$, ANTÔNIO CARLOS VIANA ${ }^{5}$
}

\author{
${ }^{1}$ Eng. Agrônomo, Doutorando em Genética e Melhoramento de Plantas da Universidade Federal de Lavras. Caixa \\ Postal 37, CEP: 37200-000 Lavras, MG. E-mail: msgomes@ufla.br (autor para correspondência) \\ ${ }^{2}$ Dr., Professor do Departamento de Agricultura da Universidade Federal de Lavras. Caixa Postal 37, CEP: $37200-000$ \\ Lavras, $M G$ \\ ${ }^{3}$ Pesquisador, Embrapa Gado de Leite. Rua Eugênio do Nascimento, 630, CEP: 36038-330 Juiz de Fora, MG \\ ${ }^{4}$ Dr., Professor do Departamento de Biologia da Universidade Federal de Lavras. Caixa Postal 37, CEP: $37200-000$ \\ Lavras, $M G$ \\ ${ }^{5}$ Pequisador, Embrapa Milho e Sorgo. Caixa Postal 151, CEP: 35701-970 Sete Lagoas, MG
}

Revista Brasileira de Milho e Sorgo, v.1, n.2, p.83-90, 2002

\begin{abstract}
RESUMO - Estudaram-se os fatores de adaptabilidade e estabilidade de cultivares de milho para caracteres associados a qualidade da silagem, em diferentes condições ambientais. Foram avaliadas dez cultivares em diferentes locais e safras agrícolas (Capinópolis, Janaúba, Lavras e Sete Lagoas, nas safras de 1997/98, 1998/99 e 1999/00, e Coronel Pacheco, nas safras de 1997/98 e 1998/99). Em todos os ambientes, o delineamento experimental utilizado foi o de blocos casualizados, com três repetições. No estádio farináceo-duro, procedeu-se à colheita das plantas da parcela, que foram cortadas e pesadas, para a determinação do peso da amostra seca ao ar. Em seguida, a matéria seca das amostras das repetições de cada cultivar foi misturada, moída e passada em peneira de $5 \mathrm{~mm}$, para a avaliação da degradabilidade in situ da matéria seca, após 24 horas de incubação no rúmem de bovino. As análises de adaptabilidade e estabilidade foram realizadas utilizando os procedimentos de Eberhart e Russel (1966) e Annicchiarico (1992). Foi estimado também um índice para seleção das cultivares, obtido a partir da multiplicação do caráter produção de matéria seca pela degradabilidade, utilizando as médias de cada ambiente. Observou-se que a interação cultivares e ambientes foi significativa apenas para o caráter produtividade de matéria seca. As cultivares avaliadas diferiram quanto à adaptabilidade e à estabilidade de produtividade de matéria seca. A metodologia de Annicchiarico (1992) discriminou as cultivares superiores quanto à produtividade de matéria seca, em que as cultivares CO 9621, DINA 1000 e DINA 657 foram as que apresentaram menor risco de baixa produtividade de matéria seca. Essas cultivares foram também as que apresentaram melhor desempenho, de acordo com o índice utilizado.
\end{abstract}

Palavras-chaves: Zea mays, qualidade da silagem, interação genótipos e ambientes.

\section{ADAPTABILITY AND STABILITY OF CORN CULTIVARS FOR DRY MATTER YIELD AND RUMINAL DEGRADABILITY OF SILAGE}

ABSTRACT - The objective of this work was to investigate the adaptability and stability
factors of corn cultivars for traits associated with silage quality under different environmental
conditions. Ten cultivars in different locations and agricultural crops (Capinópolis, Janaúba, 
Lavras and Sete Lagoas in the crops of 97/98, 98/99 and 99/00 and Coronel Pacheco in the crops of 97/98 and 98/99) were evaluated. In all the environments the experimental design utilized was that of randomized blocks with three replicates. At the hard-floury stage the harvest of the plants was proceeded and they were chopped and weighted for determining the weight of the air-dried sample. Next, the dry matter of the samples of each cultivar replicates was mixed, ground and past into a $5 \mathrm{~mm}$ screen for evaluation of the in situ degradability of dry matter after 24 hours incubation in the bovine rumen. The analyses of adaptability and stability were accomplished by utilizing the Eberhart and Russell (1966) and the Annicchiarico (1992) procedures. It was also estimated the index for selection of the cultivars, obtained from the multiplication of the trait dry matter yield by the degradability, utilizing the means of each environment. It was found that the cultivar X environment interaction was significant only for the trait dry matter yield. The cultivars evaluated differed concerning the adaptability and stability of dry matter yield. Annicchiarico's methodology (1992) distinguished the superior cultivars as to dry matter yield where the cultivars CO 9621, DINA 1000 and DINA 657 were the ones which presented the lowest risk of low dry matter yield. Those cultivars also presented the best performance according to the evaluation index utilized.

Key words: Zea mays, silage quality, genotype x environment interaction

No Estado de Minas Gerais, concentramse algumas das maiores bacias leiteiras do país. $\mathrm{Na}$ maioria dessas regiões, a principal dificuldade é o período seco do outono e inverno, que se estende dos meses de maio até setembro. A principal opção para a alimentação do rebanho nessa época é a silagem. Entre as opções de forragens para ensilagem, o milho se destaca, por apresentar grande produtividade de matéria seca, bom valor nutritivo e boa digestibilidade.

Há vários fatores que afetam a eficiência na produção de silagem utilizando a cultura do milho; entre eles, a cultivar tem grande destaque. Inúmeros relatos são encontrados na literatura sobre a diferença entre cultivares de milho com relação especificamente à produtividade de matéria seca (Melo et al., 1999; Villela, 2001). São menos freqüentes os trabalhos procurando identificar genótipos que associem produtividade de matéria seca e boa digestibilidade.

Existem relatos de que os fatores ambientais também afetam a produtividade e a qualidade da silagem (Ramalho, 1999; Fonseca, 2000; Gomes et al., 2001). Contudo, são escassos os trabalhos procurando verificar o efeito da interação dos genótipos e ambientes na expressão dos caracteres que afetam a qualidade da silagem. A presença de interação cultivares de milho e ambientes para produtividade de grãos é relatada na literatura (Gonçalves, 1997; Ribeiro et al., 2000). Nesses trabalhos, não só tem sido quantificado o efeito da interação para a expressão fenotípica, como também procurado alternativas para atenuar seu efeito.

Um estudo mais detalhado da ocorrência da interação de cultivares de milho e ambientes para os caracteres de qualidade da silagem pode melhorar a eficiência de recomendação de novas cultivares e esse foi precisamente o objetivo deste trabaho.

\section{MATERIAL E MÉTODOS}

Foram utilizados dados de experimentos de avaliação de cultivares visando a produção de silagem, gentilmente cedidos pela Embrapa Gado de Leite. Nesses experimentos, foram avaliadas 60 cultivares de milho, especialmente híbridos, em diferentes locais e durante três safras agrícolas. Nesse estudo, foram utilizados dados referentes a dez 
cultivares (Tabela 1), comuns nos diferentes locais e safras agrícolas. Os experimentos foram conduzidos nos seguintes locais do estado de Minas Gerais: Capinópolis, Janaúba, Lavras e Sete Lagoas, nas safras de 1997/98, 1998/99 e 1999/00, e Coronel Pacheco, nas safras 1997/98 e 1998/99, perfazendo um total de quatorze ambientes.

Em todos os casos, o delineamento experimental utilizado foi o de blocos casualizados, com três repetições. As parcelas foram constituídas de uma linha de sete metros. As adubações foram efetuadas de acordo com a análise do solo. $\mathrm{O}$ espaçamento utilizado foi de $90 \mathrm{~cm}$ entre linhas, com cinco plantas/metro. No estádio farináceo-duro, procedeu-se à colheita, tendo sido as plantas cortadas a $10 \mathrm{~cm}$ da superfície do solo e pesadas. Posteriormente, todas as plantas da parcela foram picadas, para a retirada de uma amostra, que foi secada em estufa de ventilação forçada, a $55^{\circ} \mathrm{C}$, até a obtenção de peso constante, e determinada a porcentagem de matéria seca por parcela, a essa temperatura. Esse valor foi utilizado posteriormente para o cálculo da produtividade de matéria seca. Em seguida, a matéria seca das amostras das repetições de cada cultivar foi misturada, moída e passada em peneira de $5 \mathrm{~mm}$, para a avaliação da degradabilidade in situ, utilizando a metodologia descrita por Pereira (1997). Essas análises foram efetuadas por meio de incubação ruminal, durante 24 horas, em quatro bovinos fistulados, utilizando-se saquinhos nos quais foram colocadas as amostras da matéria seca das plantas de cada parcela. Após serem retirados do rúmen dos animais, os saquinhos foram imediatamente colocados em água gelada, para paralisação do processo de degradação, e lavados com leve agitação, em sistema de tanque com hélice agitadora, renovando-se a água até a mesma se apresentar transparente. Depois disso, foram colocados novamente em estufa, a $55^{\circ} \mathrm{C}$, por 72 horas, e pesados em balança digital. Pela diferença de peso entre essa pesagem e a efetuada antes de incubar os materiais, foi determinada a quantidade de matéria seca desaparecida, expressa em porcentagem da matéria seca degradável no rúmen. Nesse caso, o delineamento foi de blocos casualizados, com quatro repetições - quatro animais fistulados.

TABELA 1. Características dos híbridos utilizados.

\begin{tabular}{lllll}
\hline Cultivar & Empresa & Tipo & Ciclo & Grão \\
\hline AG 5011 & Agroceres/Monsanto & H. triplo & Precoce & Semidentado \\
C0 9621 & Dow Agroscience & H. triplo & Superprecoce & Semidentado \\
DINA 1000 & Dow Agroscience & H. simples modif. & Superprecoce & Semiduro \\
DINA 657 & Dow Agroscience & H. simples modif. & Superprecoce & Semiduro \\
DINA 766 & Dow Agroscience & H. simples modif. & Superprecoce & Semiduro \\
P 3021 & Pioneer & H. triplo & Precoce & Semiduro \\
SHS 4040 & Sta. Helena Sementes & H. duplo & Precoce & Duro \\
SHS 5070 & Sta. Helena Sementes & H. triplo & Superprecoce & Duro \\
TRAKTOR & Syngenta & H. duplo & Precoce & Duro \\
Z 8392 & Dow Agroscience & H. simples & Precoce & Semiduro \\
\hline
\end{tabular}


Os dados assim obtidos para produtividade de matéria seca e degradabilidade foram submetidos à análise de variância por ambiente. Após verificada a homogeneidade da variância residual, procedeu-se à análise de variância conjunta, envolvendo todos os ambientes (Ramalho, Ferreira e Oliveira, 2001). A partir dos resultados médios, foram estimados os parâmetros que avaliam a adaptabilidade e estabilidade, utilizando-se o procedimento de Eberhart \& Russel (1966), de acordo com o seguinte modelo:

$\mathrm{Y}_{\mathrm{ij}}=\beta_{0 \mathrm{i}}+\beta_{\mathrm{li}} \mathrm{I}_{\mathrm{j}}+\delta_{\mathrm{ij}}+\varepsilon_{\mathrm{ij},}$

Emque:

$\mathrm{Y}_{\mathrm{ij}}$ : valor observado do genótipo i, no ambiente j e na repetição $\mathrm{k}$;

$\beta_{0:}:$ média geral do genótipo i;

$\beta_{1 \mathrm{i}}$ : resposta linear do genótipo i a variação ambiental;

$\mathrm{I}_{\mathrm{j}}$ : índice ambiental (diferença entre a média de cada ambiente e a média geral);

$\delta_{\mathrm{ij}}$ : desvio da regressão do genótipo i no ambiente j; $\varepsilon_{\mathrm{ij}}$ : erro experimental médio.

Foi estimado também o risco de adoção de uma determinada cultivar, utilizando o procedimento de Annicchiarico (1992). Para isto, foi estimada a percentagem $\left(\mathrm{Y}_{\mathrm{i}}\right)$ de cada cultivar em relação à média dos ambientes. Posteriormente, foi estimado o desvio-padrão fenotípico percentual $\left(\mathrm{s}_{\mathrm{i}}\right)$ de cada cultivar. A partir desse dados, foi estimado o índice de confiança $\left(\mathrm{W}_{\mathrm{i}}\right)$, pela expressão:

$\mathrm{W}_{\mathrm{i}}=\mathrm{Y}_{\mathrm{i} .}-\mathrm{Z}_{(1-\alpha)} \mathrm{s}_{\mathrm{i}}$

Emque:

$\mathrm{W}_{\mathrm{i}}$ : índice de confiança (\%);

$\mathrm{Y}_{\mathrm{i}}$ : média geral do genótipo i em porcentagem;

$Z$ : percentil $(1-\alpha)$ da funcão de distribuição normal acumulada;

$\alpha$ : nivel de significância;

$\mathrm{s}_{\mathrm{i}}$ : desvio-padrão dos valores percentuais.

A partir dos dados médios de produtividade de matéria seca e degradabilidade obtidos nos oito locais onde foram realizadas as duas avaliações, estimou-se um índice, resultado da multiplicação da característica produtividade de matéria seca pela degradabilidade. A partir daí, foi realizada uma análise de variância para esse índice, que corresponde à produtividade de matéria seca degradável por hectare, considerando cada ambiente como sendo uma repetição.

\section{RESULTADOS E DISCUSSÃO}

A acurácia experimental, avaliada pelo coeficiente de variação $(\mathrm{CV})$, variou entre as características estudadas. Observou-se um maior $\mathrm{CV}$ para a produtividade de matéria seca $(17,22 \%)$ do que para a degradabilidade (12,27\%). Vale salientar que, no caso da degradabilidade, foram utilizados dados de oito dos quatorze ambientes envolvidos. Para esse caráter, apenas a fonte de variação ambiente foi significativa $(\mathrm{P}<0,01)$. No caso da produtividade de matéria seca, todas as fontes de variação foram significativas $(\mathrm{P}<0,01)$.

Constata-se, na Tabela 2, que a degradabilidade média variou de 54,4\%, em Lavras/safra 1997/98, a 43,8\%, em Lavras/safra 1998/99. A variação para esse caráter ocorreu entre locais e até mesmo entre anos, dentro do mesmo local. A amplitude de variação para a produtividade de matéria seca foi ainda maior. Ela variou de 10,2 t ha-1, em Sete Lagoas 1997/98, a 22,0 t ha ${ }^{-1}$, em Lavras 1998/99. Aqui também a variação ocorreu tanto entre locais como entre safras, dentro de um mesmo local.

Como já mencionado, a interação cultivares e ambientes só foi detectada para a produtividade de matéria seca. A existência dessa interação, para esse caráter, já foi constatada em outros trabalhos realizados na região (Ramalho, 1999; Fonseca, 2000; Gomes et al., 2001). É oportuno salientar que a interação contribuiu com $18,96 \%$ da variação total, ao passo que o efeito de ambientes participou com $49,2 \%$ e cultivares, com apenas $6,2 \%$. 
TABELA 2. Produtividade média de matéria seca (MS) e degradabilidade (DEG) de cultivares de milho em diferentes ambientes do estado de Minas Gerais ${ }^{1}$.

\begin{tabular}{lcc}
\hline Ambientes & MS $\left(\mathrm{t} \mathrm{ha}^{-1}\right)$ & DEG (\%) \\
\hline Capinópolis - 1997/98 & $15,2 \mathrm{c}$ & \\
Cel. Pacheco - 1997/98 & $15,2 \mathrm{c}$ & $51,1 \mathrm{~b}$ \\
Janaúba - 1997/98 & $17,9 \mathrm{~b}$ & $45,2 \mathrm{~d}$ \\
Lavras - 1997/98 & $17,9 \mathrm{~b}$ & $54,4 \mathrm{a}$ \\
Sete Lagoas - 1997/98 & $10,2 \mathrm{f}$ & \\
Capinópolis - 1998/99 & $15,0 \mathrm{c}$ & \\
Cel. Pacheco - 1998/99 & $17,2 \mathrm{~b}$ & $52,8 \mathrm{a}$ \\
Janaúba - 1998/99 & $15,7 \mathrm{c}$ & \\
Lavras - 1998/99 & $22,0 \mathrm{a}$ & $43,8 \mathrm{~d}$ \\
Sete Lagoas - 1998/99 & $12,5 \mathrm{c}$ & $52,4 \mathrm{a}$ \\
Capinópolis - 1999/00 & $13,9 \mathrm{~d}$ & \\
Janaúba - 1999/00 & $16,1 \mathrm{c}$ & \\
Lavras - 1999/00 & $12,1 \mathrm{e}$ & $48,1 \mathrm{c}$ \\
Sete Lagoas - 1999/0 & $11,8 \mathrm{c}$ & $49,4 \mathrm{~b}$ \\
\hline
\end{tabular}

${ }^{1}$ Médias seguidas da mesma, letra na coluna, não diferem entre si pelo teste de Scott \& Knott (1974), a $5 \%$ de probabilidade.

A produtividade média de matéria seca variou de 13,7 t ha-1 (híbrido P 3021) a 17,2 $\mathrm{t} \mathrm{ha}^{-1}$ (híbrido CO 9621) (Tabela 3). Esses valores são semelhantes aos que têm sido relatados em experimentos de avaliação de híbridos comerciais na região (Melo et al., 1999; Fonseca, 2000; Villela, 2001).

Como ocorreu diferença significativa entre ambientes, cultivares e interação cultivares e ambientes para a produtividade de matéria seca, foi possível avaliar a adaptabilidade e estabilidade das cultivares. O primeiro problema nesse caso é o conceito de adaptabilidade a ser utilizado (Mariotti et al., 1976; Vencovsky e Barriga, 1992; Cruz e Regazzi, 1994). Quando se trata de produtividade de grãos, tem sido utilizado como mais adaptada a cultivar com maior produtividade de grãos, isto é, a que produz maior número de descendentes. No presente caso, por se tratar de matéria seca, o mesmo raciocínio não pode ser utilizado. Entretanto, podese inferir que as cultivares com maior exuberância vegetativa sejam as mais adaptadas. Assim, optouse por utilizar a produtividade média de matéria seca como medida de adaptabilidade. As cultivares mais adaptadas foram CO 9621, DINA 1000 e DINA 657.

TABELA 3. Produtividade média de matéria seca (MS) e degradabilidade (DEG) de cultivares de milho avaliadas em diferentes ambientes no estado de Minas Gerais ${ }^{1}$.

\begin{tabular}{lcc}
\hline Cultivares & MS $\left(\mathrm{t} \mathrm{ha}^{-1}\right)$ & DEG (\%) \\
\hline CO 9621 & $17,2 \mathrm{a}$ & $49,2 \mathrm{a}$ \\
DINA 1000 & $16,4 \mathrm{a}$ & $50,5 \mathrm{a}$ \\
DINA 657 & $16,1 \mathrm{a}$ & $49,4 \mathrm{a}$ \\
AG 5011 & $14,5 \mathrm{~b}$ & $50,6 \mathrm{a}$ \\
DINA 766 & $14,7 \mathrm{~b}$ & $48,8 \mathrm{a}$ \\
P 3021 & $13,7 \mathrm{~b}$ & $48,2 \mathrm{a}$ \\
SHS 4040 & $15,2 \mathrm{~b}$ & $48,8 \mathrm{a}$ \\
SHS 5070 & $14,5 \mathrm{~b}$ & $50,6 \mathrm{a}$ \\
TRAKTOR & $14,6 \mathrm{~b}$ & $49,4 \mathrm{a}$ \\
Z 8392 & $14,4 \mathrm{~b}$ & $49,4 \mathrm{a}$ \\
\hline
\end{tabular}

${ }^{1}$ Médias seguidas da mesma letra, na coluna, não diferem entre si pelo teste de Scott \& Knott (1974), a $5 \%$ de probabilidade.

Pelo procedimento preconizado por Cruz \& Regazzi (1997), para a metodologia de Eberhart \& Russel (1966), considera-se como cultivares de adaptabilidade ampla ou geral aquelas que apresentam coeficiente de regressão igual a 1 . Neste trabalho, como todos os $\beta_{1 \mathrm{i}}$ não diferiram significativamente da unidade, todas as cultivares podem ser 
consideradas como de adaptabilidade ampla ou geral (Tabela 4).

Utilizando o método que avalia o índice de confiança (Annicchiarico, 1992), tem-se a vantagem de recomendar as cultivares considerando o risco de apresentarem desempenho abaixo de um dado padrão, como, por exemplo, a média geral. Quanto maior o índice de confiança de uma cultivar, menor será a sua probabilidade de insucesso. Avaliandose as cultivares por esse método, destacaram-se novamente os híbridos CO 9621, DINA 1000 e DINA 657, que apresentaram-se com $75 \%$ de probabilidade de, na pior das hipóteses, ficarem 9,5, 4,2 e $3,9 \%$, respectivamente, acima da média dos ambientes.

TABELA 4. Estimativas de parâmetros de adaptabilidade e estabilidade de cultivares de milho, segundo a metodologia de Eberhart e Russel (1966) e índice de confiança (Wi) de Annicchiarico (1992), considerando a produtividade de matéria seca(MS), em 14 ambientes do estado de Minas Gerais.

\begin{tabular}{|c|c|c|c|c|c|}
\hline \multirow{2}{*}{ Cultivares } & \multicolumn{5}{|c|}{ MS } \\
\hline & $\beta_{0 i}$ & $\beta_{\mathrm{li}}$ & $\delta_{i j}$ & $\mathrm{R}^{2}(\%)$ & $\mathrm{W}_{\mathrm{i}}$ \\
\hline CO 9621 & $17,27 \mathrm{a}$ & $1,23^{\mathrm{ns}}$ & $3,32 * \hbar$ & 73,36 & 109,52 \\
\hline DINA 1000 & $16,49 \mathrm{a}$ & $1,10^{\mathrm{ns}}$ & $3,44 * 4$ & 68,23 & 104,22 \\
\hline DINA 657 & $16,17 \mathrm{a}$ & $1,14^{\mathrm{mat}}$ & $-0,85^{\mathrm{mx}}$ & 90,22 & 103,91 \\
\hline AG 5011 & $14,49 \mathrm{~b}$ & $0,86^{\text {15 }}$ & $2,68 *$ & 60,15 & 91,70 \\
\hline DINA 766 & $14,78 \mathrm{~b}$ & $1,02^{\mathrm{ns}}$ & $-0,85^{\mathrm{nS}}$ & 77,17 & 94,23 \\
\hline P 3021 & $13,77 \mathrm{~b}$ & $0,80^{\mathrm{ma}}$ & $4,97 * *$ & 47,29 & 86,85 \\
\hline SHS 4040 & $15,22 \mathrm{~b}$ & $1,06^{\mathrm{ns}}$ & $-1,13^{\mathrm{ns}}$ & 90,98 & 98,22 \\
\hline SHS 5070 & $14,50 \mathrm{~b}$ & $0,81^{\mathrm{ns}}$ & $1,12^{\mathrm{ns}}$ & 66,14 & 92,48 \\
\hline TRAKTOR & $14,65 \mathrm{~b}$ & $1,04^{\mathrm{ns}}$ & $-0,89^{\pi 5}$ & 88,81 & 93,77 \\
\hline Z 8392 & $14,45 \mathrm{~b}$ & $0,51^{\mathrm{ma}}$ & $0,74^{\text {ta }}$ & 73,58 & 92,38 \\
\hline
\end{tabular}

Médias seguidas da mesma letra, não diferem entre si, pelo teste de Scott \& Knott (1974), a 5\% de probabilidade.

$*$ e ** Significativo a $5 \%$ e $1 \%$ de probabilidade, respectivamente, pelo teste $t$.
A estabilidade do comportamento das cultivares, avaliada pela estimativa de $\mathrm{R}^{2}$ da metodologia de Eberhart \& Russel (1966), mostrou que as maiores estimativas para esse parâmetro foram obtidas com os híbridos DINA 657, SHS 4040 e TRAKTOR. Considerando, em termos percentuais, que a estimativa do valor de $\mathrm{R}^{2}$ pode variar de 0 a $100 \%$, pode-se inferir que essas cultivares possuem comportamento bastante previsível para a produtividade de matéria seca, uma vez que elas obtiveram valores de $\mathrm{R}^{2}$ superiores a $88 \%$.

Como comentado anteriormente, para o caráter degradabilidade, não foi detectada diferença significativa entre as cultivares avaliadas e para a interação cultivares e ambientes. Apesar da não significância para essas fontes de variação, optouse por avaliar a adaptabilidade e a estabilidade das cultivares para esse caráter.

Para a degradabilidade da matéria seca, foi utilizado o procedimento preconizado por Cruz e Regazzi (1997), para a metodologia de Eberhart \& Russel (1966), pela qual cultivares que apresentam coeficiente de regressão igual a 1,0 são consideradas de adaptabilidade ampla ou geral. Como todos os $\beta_{1 \mathrm{i}}$ não diferiram significativamente da unidade, todas as cultivares podem ser consideradas como de adaptabilidade ampla ou geral para o caráter em questão (Tabela 5).

Para a estabilidade das cultivares, quanto à degradabilidade, avaliada pela estimativa de $\mathrm{R}^{2} \mathrm{da}$ metodologia de Eberhart \& Russel (1966), chamam a atenção as baixas estimativas para esse parâmetro, $32,65 \%$ e $51,73 \%$, obtidas com as cultivares CO 9621 e P 3021, respectivamente, sendo, portanto, esses materiais os de menor previsibilidade. Com relação às demais cultivares, as diferenças não foram tão acentuadas, sendo o híbrido AG 5011, com $\mathrm{R}^{2}$ de $88,9 \%$, a cultivar com degradabilidade de matéria seca mais previsível. Esse mesmo híbrido também apresentou a maior estimativa do índice 
de confiança $\left(\mathrm{W}_{\mathrm{i}}\right)$, obtido pela metodologia de Annicchiarico (1992), ou seja, o híbrido AG 5011, mesmo com $75 \%$ de probabilidade, ficou, na pior das hipóteses, 1,4\% acima da média dos ambientes. No extremo oposto, utilizando a mesma metodologia, a cultivar P 3021 terá desempenho para degradabilidade de $4,66 \%$ abaixo da média dos ambientes, no nível de probabilidade considerado (Tabela 5).

TABELA 5. Estimativas de parâmetros de adaptabilidade e estabilidade de cultivares de milho segundo o modelo de Eberhart e Russel (1966) e índice de confiança (Wi) de Annicchiarico (1992), considerando a degradabilidade de matéria seca (DEG), em oito ambientes no estado de Minas Gerais.

\begin{tabular}{lccccc}
\hline \multirow{2}{*}{ Cultivares } & \multicolumn{5}{c}{ DEG } \\
\cline { 2 - 6 } & $\beta_{0 \mathrm{i}}$ & $\beta_{\mathrm{ii}}$ & $\delta_{i j}$ & $\mathrm{R}^{2}(\%)$ & $\mathrm{W}_{\mathrm{i}}$ \\
\hline AG 5011 & $50,66 \mathrm{a}$ & $0,68^{\mathrm{ns}}$ & $-8,31$ & 88,85 & 101,35 \\
CO 9621 & $49,21 \mathrm{a}$ & $0,66^{\mathrm{ns}}$ & 5,98 & 32,65 & 97,21 \\
DINA 1000 & $50,57 \mathrm{a}$ & $1,29^{\mathrm{m}}$ & 0,19 & 74,54 & 100,04 \\
DINA 657 & $49,45 \mathrm{a}$ & $0,87^{\mathrm{ns}}$ & $-4,35$ & 71,82 & 98,55 \\
DINA 766 & $48,80 \mathrm{a}$ & $1,05^{\mathrm{ns}}$ & $-3,38$ & 75,66 & 99,98 \\
P 3021 & $48,25 \mathrm{a}$ & $0,96^{\mathrm{m}}$ & 4,96 & 51,73 & 95,34 \\
SHS 4040 & $48,83 \mathrm{a}$ & $0,94^{\mathrm{ns}}$ & $-3,20$ & 71,00 & 97,07 \\
SHS 5070 & $50,60 \mathrm{a}$ & $1,10^{\mathrm{ns}}$ & $-5,19$ & 83,29 & 100,83 \\
TRAKTOR & $49,44 \mathrm{a}$ & $1,17^{\mathrm{ns}}$ & $-1,86$ & 75,63 & 98,17 \\
Z 8392 & $50,56 \mathrm{a}$ & $1,23^{\mathrm{mi}}$ & $-4,65$ & 84,57 & 100,55 \\
\hline
\end{tabular}

Médias seguidas da mesma letra não diferem entre si, pelo teste de Scott \& Knott (1974), a 5\% de probabilidade.

$*$ e ** Significativo a $5 \%$ e $1 \%$ de probabilidade, respectivamente pelo teste $\mathrm{t}$.

Considerando que uma cultivar ideal para a produção de silagem seja aquela que associe alta produtividade de matéria seca e maior degradabilidade possível, utilizaram-se os dados médios desses caracteres para estimar um índice que corresponde à produtividade de matéria seca degradável por hectare.
A partir dos dados desse índice, foi realizada uma análise de variância tomando cada ambiente como sendo uma repetição. Vale salientar, novamente que, no caso da degradabilidade, foram utilizados dados de apenas oito dos quatorze ambientes envolvidos. Foi verificada diferença significativa $(\mathrm{P}<$ $0,05)$ entre as cultivares avaliadas. Pelo teste de média (Tabela 6) as cultivares foram divididas em dois grupos, em que os híbridos CO 9621, DINA 1000 e DINA 657, com produtividade de matéria seca degradável por hectare acima de $8,3 \mathrm{tha}^{-1}$ foram superiores às demais cultivares testadas.

TABELA 6. Produtividade média de matéria seca degradável (MS DEG) de cultivares de milho, em oito ambientes do estado de Minas Gerais.

\begin{tabular}{lc}
\hline Cultivares & MS DEG $\left(\mathrm{t} \mathrm{ha}^{-1}\right)$ \\
\hline CO 9621 & $8,9 \mathrm{a}$ \\
DINA 1000 & $8,5 \mathrm{a}$ \\
DINA 657 & $8,3 \mathrm{a}$ \\
Z 8392 & $7,9 \mathrm{~b}$ \\
SHS 4040 & $7,8 \mathrm{~b}$ \\
AG 5011 & $7,8 \mathrm{~b}$ \\
DINA 766 & $7,6 \mathrm{~b}$ \\
TRAKTOR & $7,5 \mathrm{~b}$ \\
SHS 4040 & $7,1 \mathrm{~b}$ \\
P 3021 & $6,6 \mathrm{~b}$ \\
\hline
\end{tabular}

Médias seguidas da mesma letra não diferem entre si, pelo teste de Scott \& Knott (1974), a 10\% de probabilidade.

\section{Conclusões}

As cultivares avaliadas diferem quanto à adaptabilidade e à estabilidade de produtividade de matéria seca.

A metodologia de Annicchiarico (1992) discriminou cultivares superiores quanto à produtividade de matéria seca, em que os híbridos CO 9621, 
DINA 1000 e DINA 657 foram os que apresentaram menor risco de baixa produtividade de matéria seca. Essas cultivares foram também as que apresentaram melhor desempenho, de acordo com o índice avaliado.

\section{Literatura Citada}

ANNICCHIARICO, P. Cultivar adaptations and recommendation from alfafa trials in Northern Italy. Journal of Genetics \& Breeding, Rome, v.46, n.1, p.269-278, mar. 1992.

CRUZ, C.D.; REGAZZI, A.J. Modelos biométricos aplicados ao melhoramento genético. Viçosa: UFV, 1994. 390p.

EBERHART, S.A.; RUSSELL, W.A. Stability parameters for comparing varieties. Crop Science, Madison, v.6, n.1, p.36-40, Jan./Feb. 1966.

FONSECA, A. H. Características químicas e agronômicas associadas à degradabilidade da silagem de milho. 2000. 93f. Dissertação (Mestrado em Fitotecnia) - Universidade Federal de Lavras, Lavras.

GOMES, M.S.; VON PINHO, R.G.; OLIVEIRA, J.S.; VIANA, A.C. Avaliação de cultivares de milho para a produção de silagem: parâmetros genéticos e interação genótipos por ambientes. In: CONGRESSO BRASILEIRO DE MELHORAMENTO DE PLANTAS, 1., 2001, Goiânia. Anais... Goiânia: Embrapa Arroz e Feijão, 2001. CD-Rom (Embrapa Arroz e Feijão. Documentos, 113)

GONÇALVES, F.M.A. Adaptabilidade e estabilidade de cultivares de milho avaliadas em "safrinha" no período de 1993 a 1995. 1997. 86f. Dissertação (Mestrado em Genética e Melhoramento de Plantas) - Universidade Federal de Lavras, Lavras.

MARIOTTI, J.A.; OYARZABAL, E.S.; OSA, J.M.; BULACIO, A.N.R.; ALMADA, G.H. Análisis de estabilidad y adaptabilidad de genótipos de cana de azúcar. I.Interacciones dentro de una localidad experimental. Revista Agronómica del Noroeste Argentino, San Miguel de Tucumán, v.13, n.14, p.105-127, ene. 1976.

MELO, W.M.C.; VON PINHO, R.G.; CARVALHO, M.L.M.; VON PINHO, E.V.R. Avaliação de cultivares de milho para produção de silagem na região de Lavras-MG. Ciência e Agrotecnologia, v.23, n.1, p.31-39. 1999.

PEREIRA, M.N. Responses of lactating cows to dietary fiber from alfafa or cereal by products. 1997. 186 f. (Thesis Phylosophy Doctor) University of Wisconsin, Madison.

RAMALHO, A.R. Comportamento de famílias de meios-irmãos em diferentes épocas de semeadura visando a produção de forragem de milho. 1999. 77f. Dissertação (Mestrado em Fitotecnia) - Universidade Federal de Lavras, Lavras.

RAMALHO, M.A.P.; FERREIRA, D.F.; OLIVEIRA, A.C. de A experimentação em genética e melhoramento de plantas. Lavras: UFLA, 2000. $326 \mathrm{p}$.

RIBEIRO, P.H.E.; RAMALHO, M.A.P.; FERREIRA, D.F. Adaptabilidade e estabilidade de genótipos de milho em diferentes condições ambientais. Pesquisa Agropecuária Brasileira, Brasília, v.35, n.11, p.2213-2222, nov. 2000.

SCOTT, D.W.; KNOTT, M.A. Acluster analysis method for grouping means in the analysis of variance. Biometrics, Raleigh, v.30, p.507-512, Sept. 1974.

VENCOVSKY, R.; BARRIGA, P. Genética biométrica no fitomelhoramento. Ribeirão Preto: Sociedade Brasileira de Genética, 1992.496p.

VILLELA, T.E.A. Época de semeadura e de corte de plantas de milho para silagem. 2001. 86f. Dissertação (Mestrado em Fitotecnia) - Universidade Federal de Lavras, Lavras. 